\title{
Reverse flood routing with the inverted Muskingum storage routing scheme
}

\author{
A. D. Koussis ${ }^{1}$, K. Mazi $^{1}$, S. Lykoudis ${ }^{1}$, and A. A. Argiriou ${ }^{2}$ \\ ${ }^{1}$ Institute for Environmental Research \& Sustainable Development, National Observatory of Athens, Greece \\ ${ }^{2}$ Department of Physics, University of Patras, Greece
}

Correspondence to: A. D. Koussis (akoussis@meteo.noa.gr)

Received: 26 January 2011 - Revised: 19 October 2011 - Accepted: 22 November 2011 - Published: 20 January 2012

\begin{abstract}
This work treats reverse flood routing aiming at signal identification: inflows are inferred from observed outflows by orienting the Muskingum scheme against the wave propagation direction. Routing against the wave propagation is an ill-posed, inverse problem (small errors amplify, leading to large spurious responses); therefore, the reverse solution must be smoothness-constrained towards stability and uniqueness (regularised). Theoretical constrains on the coefficients of the reverse routing scheme assist in error control, but optimal grids are derived by numerical experimentation. Exact solutions of the convection-diffusion equation, for a single and a composite wave, are reverse-routed and in both instances the wave is backtracked well for a range of grid parameters. In the arduous test of a square pulse, the result is comparable to those of more complex methods. Seeding outflow data with random errors enhances instability; to cope with the spurious oscillations, the reversed solution is conditioned by smoothing via low-pass filtering or optimisation. Good-quality inflow hydrographs are recovered with either smoothing treatment, yet the computationally demanding optimisation is superior. Finally, the reverse Muskingum routing method is compared to a reverse-solution method of the St. Venant equations of flood wave motion and is found to perform equally well, at a fraction of the computing effort. This study leads us to conclude that the efficiently attained good inflow identification rests on the simplicity of the Muskingum reverse routing scheme that endows it with numerical robustness.
\end{abstract}

\section{Character of the reverse routing problem}

The forward calculation of the propagation of a flood wave in an open channel, known as flood routing, is a problem of applied hydrology that has been studied extensively. The relevant methods of solution, all within the framework of one-dimensional free-surface flow, span the spectrum from numerical solutions of the hydraulic equations of Barré de Saint-Venant to storage routing models of the diffusion-wave type (Koussis, 2009), and have utility in such applications as flood warning, river training and urban storm drainage design. On occasion, however, flood related questions are posed in the reverse sense, such as, e.g., in signal identification (hydrologic forensics): "Which inflow created the outflow observed at cross-section X, or the observed flood profile along reach Y?" We may be also interested in operating a reservoir (optimal outflow control) to minimise downstream flood damage (Szöllósi - Nagy, 1987). Bruen and Dooge (2007) point out that reliable solution techniques of the latter problem would be valuable in handling of urban flash flooding.

Yet reverse routing is an inverse problem and as such not well posed. A problem is well-posed when its solution exists, is unique and stable (Bronstein and Semendjajew, 1964), that is, small changes in the initial condition (forcing) cause small changes in the response. The reverse routing solution clearly exists, but must be constrained for stability by a smoothness condition, which however does not ensure uniqueness. This is readily seen when attempting to solve a diffusion equation in reverse time: back-stepping is equivalent to calculating forward with a negative diffusion coefficient. The consequence is that errors, either present in the initial data or incurred in the computation (e.g., due to finite machine precision - rounding errors) are amplified instead of reduced by positive diffusive spreading. This manifestation of the irreversibility of diffusion, or of diffusion-like processes such as dispersion, must be considered in developing a back-stepping method.

The main features of flood wave motion are translation and attenuation, the latter due to (hydraulic) wave diffusion; both are nonlinear features resulting in nonlinear deformation. The linear convection - diffusion equation (CDE), 
stated in terms of the discharge $q$ (Lighthill and Whitham, 1955; Dooge, 1973), is the simplest physically grounded flood wave model that describes wave translation and attenuation well, and is important because its range of validity is wide (Perumal and Sahoo, 2007):

$\frac{\partial q}{\partial t}+c_{k} \frac{\partial q}{\partial x}=D \frac{\partial^{2} q}{\partial x^{2}}$

in which $x$ is the thalweg distance and $t$ is time. The constant parameters, kinematic wave celerity $c_{k}$ and coefficient of hydraulic diffusion $D$, in a channel of width $B_{o} \approx$ const., are referenced to a discharge $q_{o}$, at which the Froude number is $\mathbf{F}_{\mathbf{0}}=\left(q_{o}^{2} B_{o} / g A_{o}^{3}\right)^{1 / 2} ; A$ is the flow area, a function of the depth $y, g$ the gravitational acceleration and $S_{o}$ the bed slope:

$c_{k}=\left.\frac{d q}{d A}\right|_{x=\text { const. }}=\left.\frac{1}{B_{o}} \frac{d q}{d y}\right|_{x=\text { const. }}$

$D=\frac{q_{o}}{2 B_{o} S_{o}}\left(1-p^{2} \mathbf{F}_{\mathbf{o}}^{2}\right)$

$p$ depends on the flow formula; $p=2 / 3$ for Manning's formula, $q_{o}=(1 / n) A_{o} y_{o}^{2 / 3} S_{o}^{1 / 2}$ for wide channels, where $n$ is related to the bed roughness, and commonly $p^{2} \mathbf{F}_{\mathbf{0}}{ }^{2} \ll 1$.

Realising that the same CDE-type describes the propagation of diffusion flood waves and one-dimensional mass transport (Koussis, 1983), we can apply the method advanced here also to contaminant transport in streams, often modelled as an one-dimensional advection-dispersion problem (Fischer et al., 1979). The solute mass is transported by advection - the contaminant movement by the mean flow velocity $u$ (equivalent to the kinematic wave $[\mathrm{KW}]$ motion) and by dispersion (longitudinal dispersion coefficient $D_{L}$ ) the mathematical equivalent to hydraulic wave diffusion, but due to differential advection of fluid parcels. The mass transport CDE is written in terms of the concentration $C$ [e.g., in $\mathrm{mg} / L]$, in the place of the volumetric flow rate $q$.

Source signal identification is a forensic activity of a regulatory agency, and may also interest the courts. For example, a regulator may wish to recover the input signal from a source suspected to have caused an observed pollution incident. The recovery entails reverse computation of mass transport to the source by stepping back in time. Dispersion can be appreciable under ordinary transient loadings and is important in spills (e.g., Li, 1972; Koussis et al., 1990). The StreeterPhelps textbook-solution of the BOD-DO problem considers only the dominant advection for computational convenience. Taking into account the correspondences $q \rightarrow C, c_{k} \rightarrow u$ and $D \rightarrow D_{L}$, the reverse flood routing methodology presented in the sequel applies also to the challenging inverse problem of source signal identification in streams, assuming that advection dominates mass transport.

\section{Formulation of the reverse routing problem}

We treat the signal identification problem on the basis of the diffusion-wave model described by Eqs. (1)-(3), using a reverse Muskingum routing scheme. This reverse routing approach is tried for the first time. The Muskingum scheme approximates the propagation of the diffusion wave efficiently when the kinematic wave mode dominates; Lighthill and Whitham (1955) showed that the main flood body travels as a KW. The routing scheme derives from a first-order accurate finite difference (FD) discretisation of the KW equation and becomes a second-order accurate diffusion-wave propagation solver by matching the numerical diffusion coefficient of the KW equation solution scheme to the hydraulic diffusion coefficient of the CDE (matched artificial diffusion MAD).

Exact solution reversal is possible when the propagation is strictly kinematic. Hence, a numerical scheme that originates in the $\mathrm{KW}$ equation yet allows for numerical diffusion that matches the physical diffusion constitutes a promising basis for reversing the wave propagation computationally. First Cunge (1969) analysed such a scheme for flood routing; Koussis $(1975,1978,2009,2010)$ also linked storage routing to the diffusion-wave model. The good performance of Matched Artificial Diffusion (MAD) schemes in forward routing has been verified in flood [e.g., Koussis, 1975; Weinmann, 1977; Perkins and Koussis, 1996] and in pollution routing in streams (Koussis et al., 1983; Koussis et al., 1990). The same holds for the MAD-based solution component of longitudinal transport in groundwater (Syriopoulou and Koussis, 1991; García-Delgado and Koussis, 1997; Koussis et al., 2003).

In contrast to the rarely studied reverse flood routing problem (Bruen and Dooge, 2007), the related source signal identification problem of mass transport in groundwater has been studied (in one dimension, at first) along several lines (Michalak and Kitanidis, 2004). Early attempts placed the solution in the framework of function fitting (parameter estimation of an assumed source function). Later, however, researchers used various methods to solve this reverse problem: Skaggs and Kabala $(1994,1998)$ and Liu and Ball (1999) used the complete (deterministic) estimation method of Tikhonov regularisation, Woodbury and Ulrich $(1996,1998)$ the (stochastic) minimum relative entropy method, and Snodgrass and Kitanidis (1997) geostatistics; Neupauer et al. (2000) compared the Tikhonov regularisation and minimum relative entropy methods. The quasireversibility method of Skaggs and Kabala (1995) gave unsatisfactory results; Atmadja and Bogtzoglou (2001) solved the transport equation in reverse time with the backward beam equation. Skaggs and Kabala (1998) studied the limitations in recovering source signal information in relation to the transport parameters, the accuracy of field data and the time of plume evolution. 


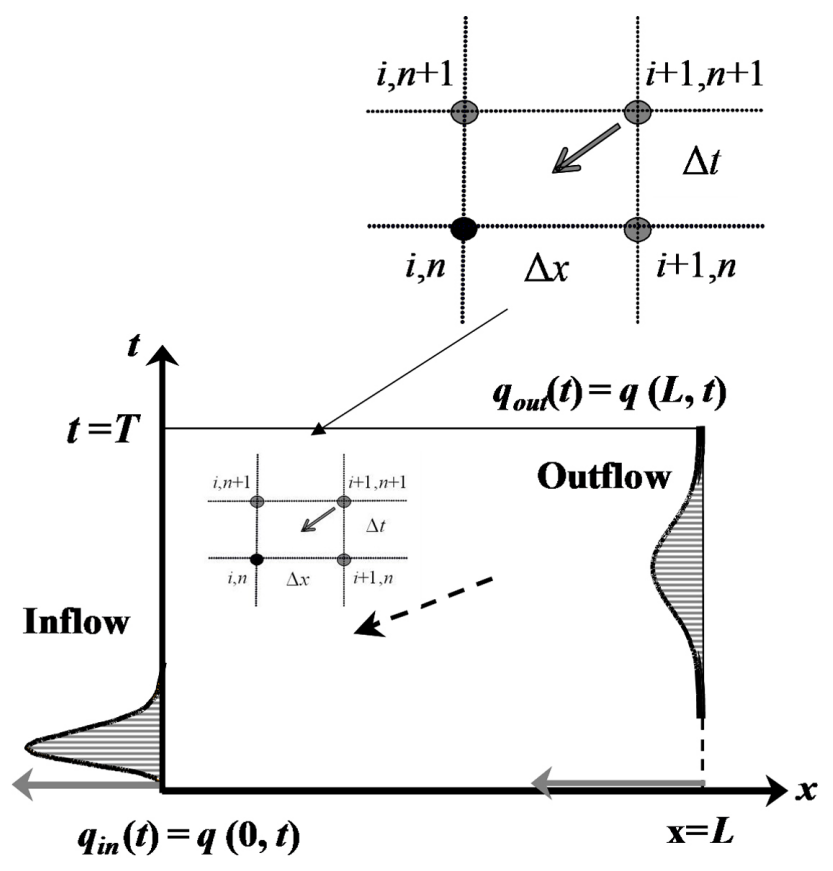

Fig. 1. Reverse flood routing in a stream reach of length $L$ : $q_{\text {out }}(t)=q(L, t)$ observed outflow hydrograph, $q_{\text {in }}(t)=q(0, t)$ inflow hydrograph; $\longrightarrow$ direction of reverse routing.

The proposed method is conceptually and computationally much simpler than the aforementioned ones. It tackles reverse routing by solving the CDE (1) indirectly, yet explicitly in reverse time, on the premise of kinematic-dominated wave propagation. Figure 1 shows the concept of identifying the inflow hydrograph $q_{\text {in }}(t)=q(0, t)$ from an observed outflow hydrograph at $x=L, q_{\text {out }}(t)=q(L, t)$. Note that, in the reverse mass transport problem, a field profile $C_{\text {field }}(x, T)$ may often be the initial condition at time $T$. In reverse flood routing, this corresponds to a flow profile $q_{\text {field }}(x, T)$ in a reach of interest; the proposed method can be used to solve this problem as well.

We begin with a reprise of the forward solution. The Muskingum-Cunge flood routing scheme (Cunge, 1969) is obtained by considering pure KW propagation, described by Eq. (1) with $D=0$. Discretising the KW equation only in space, over a grid element $\Delta x=x_{i+1}-x_{i}$, and using the spatial weighting factor $\theta$ to position the temporal derivative in $\Delta x$, Fig. 2, yields the ordinary differential equation (method of lines)

$\left.\theta \frac{d q}{d t}\right|_{i}+\left.(1-\theta) \frac{d q}{d t}\right|_{i+1}+\frac{c_{k}}{\Delta x}\left[q_{i+1}(t)-q_{i}(t)\right] \approx 0$.

Then, setting $d q / d t \approx\left(q_{n+1}-q_{n}\right) / \Delta t$ and centring the spatial difference in $\Delta t=t_{n+1}-t_{n}$ give

$\theta \frac{q\left(x_{i}, t_{n+1}\right)-q\left(x_{i}, t_{n}\right)}{\Delta t}+(1-\theta) \frac{q\left(x_{i+1}, t_{n+1}\right)-q\left(x_{i+1}, t_{n}\right)}{\Delta t}+$

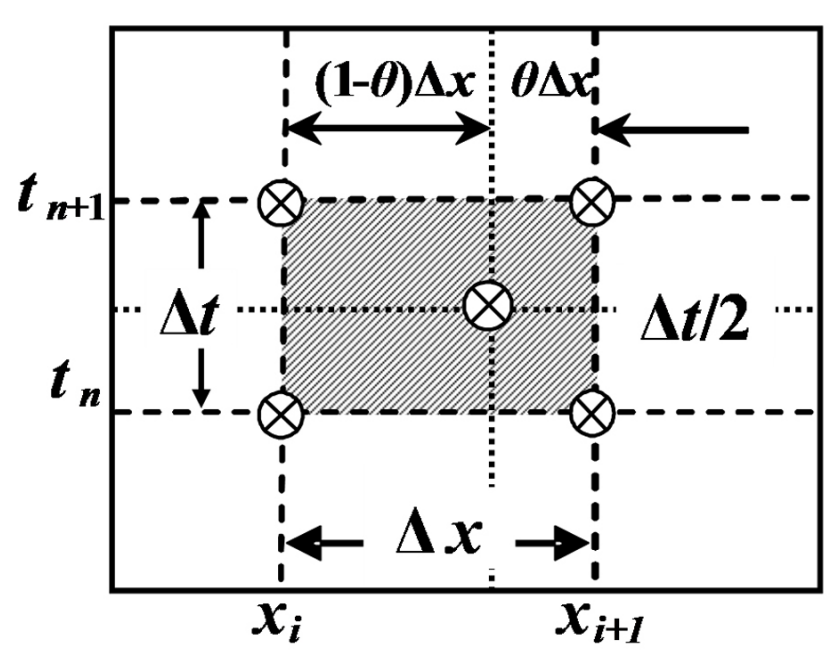

Fig. 2. Grid element for the discretisation of the KW equation and the routing calculations.

$c_{k} \frac{q\left(x_{i+1}, t_{n}\right)-q\left(x_{i+1}, t_{n+1}\right)}{2 \Delta x}+$

$c_{k} \frac{q\left(x_{i+1}, t_{n+1}\right)-q\left(x_{i}, t_{n+1}\right)}{2 \Delta x} \approx 0$.

After collecting terms, we obtain the following wellknown Muskingum FD scheme:

$q[(i+1) \Delta x,(n+1) \Delta t]=\mathrm{a}_{1} q(i \Delta x, n \Delta t)+$

$\mathrm{a}_{2} q(i \Delta x,(n+1) \Delta t)+\mathrm{a}_{3} q((i+1) \Delta x, n \Delta t)$

$\mathrm{a}_{1}=\frac{\mathbf{C}+2 \theta}{\mathbf{C}+2-2 \theta} ; \mathrm{a}_{2}=\frac{\mathbf{C}-2 \theta}{\mathbf{C}+2-2 \theta} ; \mathrm{a}_{3}=\frac{-\mathbf{C}+2-2 \theta}{\mathbf{C}+2-2 \theta}$

The $\mathrm{a}_{i}{ }^{\prime} \mathrm{s}$ in Eqs. (6) and (7) depend on the weighting factor $\theta$ and the Courant number

$\mathbf{C}=c_{k} \Delta t / \Delta x$

The expansion of the grid functions in Eq. (4) in Taylor series around $x_{i}=i \Delta x, t_{n}=n \Delta t$ to second order yields the CDE Eq. (9), when second derivatives appearing in these expansions are expressed as second spatial derivatives via the KW equation, $\frac{\partial q}{\partial t}+c_{k} \frac{\partial q}{\partial x}=0$, i.e., $\frac{\partial^{2} q}{\partial x \partial t}=-c_{k} \frac{\partial^{2} q}{\partial x^{2}}, \frac{\partial^{2} q}{\partial t^{2}}=$ $c_{k}^{2} \frac{\partial^{2} q}{\partial x^{2}}$ :

$\frac{\partial q}{\partial t}+u \frac{\partial q}{\partial x}=D_{N} \frac{\partial^{2} q}{\partial x^{2}}+$ remainder

$D_{N}$ is a numerical diffusion coefficient that is linked to the spatial discretisation:

$D_{N}=c_{k} \Delta x(0.5-\theta)$, or $\theta=0.5\left(D_{N} / c_{k} \Delta x\right)$. 
Setting $D_{N}=D$ in Eqs. (10) relates $\theta$ to the grid Peclet number, Eq. (11), as follows:

$\mathbf{P}=c_{k} \Delta x / D$,

$\theta=0.5-\left(D / c_{k} \Delta x\right)=0.5-\mathbf{P}^{-1}$.

Thus, the explicit Eq. (6) (with Eqs. 7-8) solves a CDE with second-order accuracy, provided that expressing the mixed and temporal second derivatives via the spatial one is sufficiently accurate (near-KW mode). This scheme is unconditionally stable for physically realisable cases, i.e., for $D>0(\theta<0.5) ; \theta=0.5, \mathbf{C}=1$ give exact $\mathrm{KW}$ motion. The leading term of the remainder in Eq. (9) is (Cunge, 1969):

$R=\frac{c_{k} \Delta x^{2}}{2}\left[-\frac{\mathbf{C}^{2}}{6}-\left(\frac{1}{2}-\theta\right) \mathbf{C}+\frac{2}{3}-\theta\right]\left|\frac{\partial^{3} q}{\partial x^{3}}\right|$

From Eq. (6), we develop the explicit reverse routing scheme for $q\left(x_{i}, t_{n}\right)$ :

$q(i \Delta x, n \Delta t)=b_{1} q((i+1) \Delta x,(n+1) \Delta t)+$

$b_{2} q(i \Delta x,(n+1) \Delta t)+b_{3} q((i+1) \Delta x, n \Delta t)$

The $b_{i}{ }^{\prime}$ s are obviously related to the $\mathrm{a}_{i}{ }^{\prime} \mathrm{s}$ of Eq. (7) and written as follows:

$b_{1}=\frac{\mathbf{C}+2(1-\theta)}{\mathbf{C}+2 \theta} ; b_{2}=\frac{-\mathbf{C}+2 \theta}{\mathbf{C}+2 \theta} ; b_{3}=\frac{\mathbf{C}-2(1-\theta)}{\mathbf{C}+2 \theta}$

For $0.5>\theta \geq 0$, Eq. (15) yields: $b_{1} \geq 1$, and $1>b_{2} \geq 0$ for $\mathbf{C} \leq 2 \theta$, hence $\mathbf{C} \leq 1 ; b_{3}<0$, but less so as $\mathbf{C} \rightarrow 1$ and $\theta \rightarrow 0.5$. Negative coefficients cause spurious oscillations and should be avoided if possible, or be kept small.

Given observations of the discharge (or of the stage, to be converted to flow via a rating curve) at the cross-section at $x=L, q_{\text {out }}(t)=q(L, 0 \leq t \leq T)$ (outflow) and the condition $q(L, t \geq T)=q_{\text {base }}$, the inflow (source signal) $q_{\text {in }}(t)$ can be, in principle, recovered by sequential application of Eq. (14) on the outflow data. As shown in Fig. 1, the reverse computation marches from $t=T, x=L$ to $t=0, x=0$. Yet, Eq. (14) is inherently unstable to disturbances for $\theta<0.5$. Prior to devising stabilising mechanisms, however, we study the sensitivity of reverse integration, because suitable grid parameters in Eq. (14) help error control. In theory, grid design minimises the leading remainder term $R$ that is responsible for numerical dispersion (phase errors, not attenuation). $R$ vanishes at $\mathbf{C}=1$ for all $\theta$, but this theoretically optimal condition does not hold strictly, for the scheme is based on the approximate conversion of $\partial^{2} q / \partial t^{2}$ and $\partial^{2} q / \partial x \partial t$ to $\partial^{2} q / \partial x^{2}$ via the KW equation. The numerical tests of Cunge (1969) reveal a similar behaviour for the forward solution.

Obviously, the duration of the inflow hydrograph is shorter than that of the outflow hydrograph (see space-time domain in Fig. 1). Exploiting this fact, we carry out the reverse calculations only inside the domain delimited by the KW characteristics passing through the endpoints of the outflow hydrograph. The aim of this restriction is control of spurious, troublesome oscillations that a recovered inflow can contain, not computational efficiency. Indeed, this measure largely eliminates unphysical tail oscillations of the source signal.

\section{Grid Design: Tests with perfect measurements}

We investigate optimal grid design through reverse-time tests, with $c_{k}=1$ and $D=1, V=30$ (loading) and space and time scales 600 and 300, respectively (consistent units), as used by Skaggs and Kabala $(1994,1998)$. The solution for a Dirac pulse of content $V$ released at $x_{o}$ outside the domain is (Kreft and Zuber, 1978; Szymkiewicz, 2002)

$q(x, t)=\frac{V x}{\left(4 \pi D t^{3}\right)^{0.5}} \exp \left(-\frac{\left[\left(x-x_{o}\right)-c_{k} t\right]^{2}}{4 D t}\right)$

The test grids covered the range $0<\mathbf{C} \leq 1.2,0 \leq \theta(\mathbf{P}) \leq$ 0.5 . Volume conservation and form fidelity were deemed paramount for judging a solution; therefore the ranking of the recovered inflow signals was based on the bias error measure $\mathrm{E}_{\mathrm{M}}$, Eq. (17), (in all tests, mass was conserved with $\mathrm{E}_{\mathrm{M}}<0.002$, or $0.2 \%$ ) and on the shape criterion $r$, Eq. (18):

$\mathrm{E}_{\mathrm{M}}=\mid$ cumulative volume difference between recovered and analytic inflow $\mid / \mathrm{V}$

$\mathrm{r}=($ root - mean - squared - error of recovered inflow) $/(\sigma$ of analytic inflow $)$

The tests confirm the anticipated result that $\mathbf{C}_{\text {optimal }} \neq 1$; indeed, propagation is less KW-like as $\theta \rightarrow 0$ and $\mathbf{C}_{\text {optimal }}$ deviates more from 1. According to Eq. (13), $R$ decreases as $\theta(\mathbf{P})$ increases, indicating improved accuracy with stronger $\mathrm{KW}$ behaviour. The dots of optimal $\mathbf{C}$ vs. $\theta(\mathbf{P})$ pairs shown in Fig. 3 form a soft upper limit of $\mathbf{C}$, while the $\theta$-values should not be less than $\approx 0.25$; results for $\theta<0.25$ are quasistable (oscillations are contained only for $\mathbf{C}>2$ ), but poor. The optimal $\mathbf{C}[\theta(\mathbf{P})]$-curve confirms approximately the limiting condition on $b_{2}, \mathbf{C}<2 \theta$, only up to $\theta \approx 1 / 3$; in the range $1 / 4 \leq \theta \leq 1 / 3$ the optimal $\mathbf{C}[\theta(\mathbf{P})]$-curve is steeper; of course, at the KW-point holds exactly $\mathbf{C}=1, \theta=0.5$ $(\mathbf{P} \rightarrow \infty)$. As a rule of thumb for good grid design, one may use the best-fit relation $\mathbf{C}=19.27 \theta^{2}+17.57 \theta-3.04$ in the range $0.25 \leq \theta \leq 0.45(\theta=0.45 \rightarrow \mathbf{P}=20)$.

More or less strict grid design is required depending on the scale of the problem and on the content of the pulse (Skaggs and Kabala, 1998), because the information of the field data that is useable for signal recovery diminishes as diffusion/dispersion progresses.

Next, we test these grid design rules on similar problems, but with wave parameters $c_{k}=1 \mathrm{~m} \mathrm{~s}^{-1}$ and $D=$ $1000 \mathrm{~m}^{2} \mathrm{~s}^{-1}$, which correspond to a channel of rectangular cross-section of width $B \approx 61 \mathrm{~m}$ slope $S_{o} \approx 4 \times 10^{-4}$ and Manning's $n=0.04$, so that at a mean uniform flow $q_{o}=50 \mathrm{~m}^{3} \mathrm{~s}^{-1}$ the depth is $y_{o} \approx 1.36 \mathrm{~m}$. We generate the 


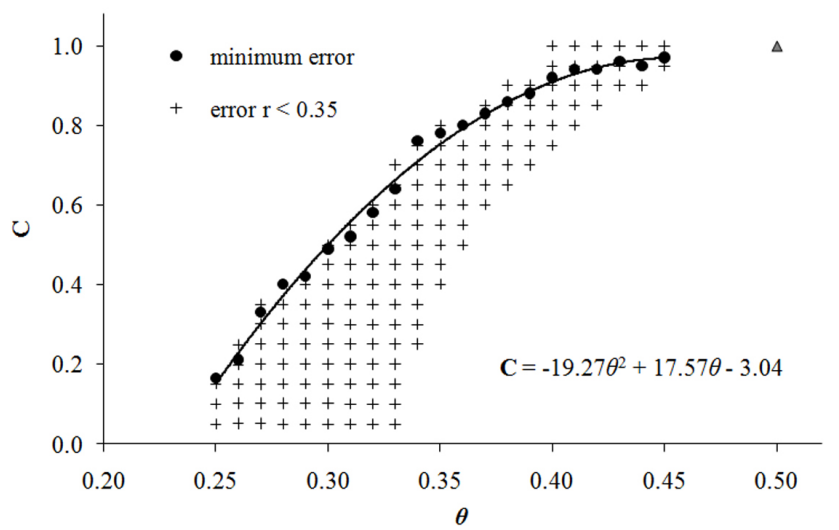

Fig. 3. Grid design for reverse routing test problem: the dark dots indicate optimal design; the stippled area +++ indicates grids satisfying the modest shape accuracy criterion $r<0.3$.

required data by releasing a Dirac pulse of volume $V=$ $5 \times 10^{6} \mathrm{~m}^{3}$ outside the domain at $x=x_{o}=-200 \mathrm{~km}, t=0$, which has the advantage of yielding an analytical solution - Eq. (16) - without convolution. These data are an inflow wave (source signal at $x=0$ ), Eq. (19), and nominally perfect field measurements at $x=L=200 \mathrm{~km}$, i.e., $200 \mathrm{~km}$ downstream, Eq. (20):

$$
\begin{array}{r}
q(0, t)=\frac{V x}{\left(4 \pi D t^{3}\right)} \exp \left[-\frac{\left(-x_{o}-c_{k} t\right)^{2}}{4 D t}\right] \\
q(L, t)=\frac{V x}{\left(4 \pi D t^{3}\right)} \exp \left[-\frac{\left[\left(L-x_{o}\right)-c_{k} t\right]^{2}}{4 D t}\right]
\end{array}
$$

The reverse scheme Eqs. (14)-(15) performs generally well with suitable grids. Figure 4 demonstrates the sensitivity of the model to the choice of $C$ and $\theta$, showing inflow signals recovered from the same outflow hydrograph using a near optimal and a non-optimal grid.

However, depending on the grid, the recovered signal can exhibit more or less pronounced spurious oscillations. It is the presence of negative coefficient(s) in Eq. (14) that causes oscillations in signal reversal, just as oscillations appear, e.g., for $a_{2}<0$, in the forward solution (scheme Eq. 6), causing the outflow dip in Muskingum flood routing. But because $a_{2}$ is not inherently negative, oscillations in the forward solution are suppressed effectively by selecting $\mathbf{C}$ and $\theta$ such that all $a_{i} \geq 0$ (e.g., Bowen et al., 1989). Indeed, this numerical artifact is removed entirely for $\theta=0$ (time derivative positioned at $x_{i+1}$ ). In the reverse solution, oscillations cannot be eliminated (at least, $b_{2}<0$ or $b_{3}<0$ ), yet can be contained (for perfect input data) by using an appropriate grid.

The spurious oscillations are explained by considering a grid element as a linear Muskingum-reservoir with time constant $\Delta x / c_{k}$. The output is obtained by convolving the system response function (SRF) with the input. The forward solution of Eq. (4), taking $q_{i}(0)=q_{i+1}(0)=0$ without loss
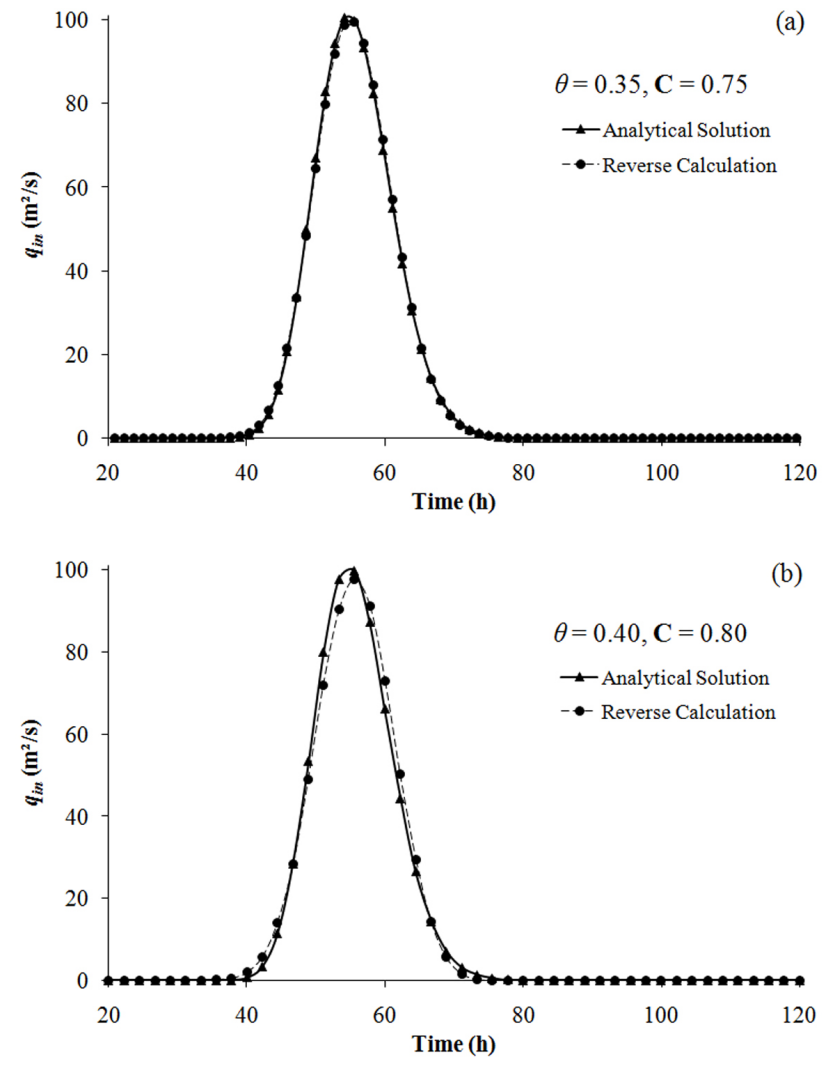

Fig. 4. Single-peak inflow hydrograph recovered through reverse routing from perfect outflow data at $x=200 \mathrm{~km}$ with grids: $C=$ $0.75, \theta=0.35$ (near-optimal) and $C=0.8, \theta=0.4$ (non-optimal); the analytical inflow is also shown for comparison.

of generality, is (Nash, 1959; Venetis, 1969)

$$
\begin{array}{r}
q=\frac{c_{k} / \Delta x}{(1-\theta)^{2}} \int_{0}^{t} q_{i}(\tau) \exp \left\{-\frac{c_{k}(t-\tau)}{\Delta x(1-\theta)}\right\} d \tau \\
-\frac{\theta}{1-\theta} q_{i}(t)
\end{array}
$$

Evidently, the negative term in Eq. (21) is the root cause of spurious oscillations for $\theta>0$; however, these oscillations can be masked numerically by using $\mathbf{C} \geq 2 \theta$ in Eqs. (6)-(7).

Similarly, formal reverse solution of Eq. (4) for $q_{i}(t)$, again for $q_{i}(0)=q_{i+1}(0)=0$, gives

$$
\begin{array}{r}
q_{i+1}=\frac{-c_{k} / \Delta x}{\theta^{2}} \int_{0}^{t} q_{i+1}(\tau) \exp \left\{-\frac{c_{k}(t-\tau)}{\Delta x(1-\theta)}\right\} d \tau \\
-\frac{1-\theta}{\theta} q_{i+1}(t)
\end{array}
$$

Reorienting the convolution integral in Eq. (22) from $T$ to $t<T$ (sign reversal conforms to a calculation that steps back in time) yields the reverse response Eq. (23): 
$q_{i+1}=\frac{c_{k} / \Delta x}{\theta^{2}} \int_{T}^{t<T} q_{i+1}(\tau) \exp \left\{-\frac{c_{k}(t-\tau)}{\Delta x(1-\theta)}\right\} d \tau$

$-\frac{1-\theta}{\theta} q_{i+1}(t)$

Alternatives to Eq. (14) can be derived from Eq. (23) by numerical evaluation of the integral (Nash, 1959; Seus and Rösl, 1972; Koussis, 1975; Koussis, 1980; Bowen et al., 1989), yet oscillations are expected again, since division by $\theta$ indicates potential severe instability as $\theta \rightarrow 0$. Indeed, our initial tests of Eqs. (14)-(15) for feasible grids showed that the accuracy in the recovery of inflow signals suffers greatly for $\theta<0.25$. Of course, the reverse KW solution (obtained for $\theta=0.5, \mathbf{C}=1$ ) is exact, as in the forward routing.

Next, we test the scheme's ability to recover more complex signals, such as from two impulses $V_{1}=5 \times 10^{6} \mathrm{~m}^{3}$ and $V_{2}=$ $2.5 \times 10^{6} \mathrm{~m}^{3}$ released, respectively, at $x_{o 1}=-200 \mathrm{~km}$ and at $x_{o 2}=-275 \mathrm{~km}$, at $t=0$ (obtained by twice-superposing Eq. 16); the observations are made again at $x=200 \mathrm{~km}$. The results of reverse routing are shown in Fig. 5.

\section{Reverse routing with imperfect measurements}

So far, the outflow data have been assumed perfect; however, measurement errors make always field data inexact. For this reason, the performance of the reverse scheme Eqs. (14) (15) is tested also with error-seeded data. The nominal discrete field data $q_{\text {out }}(t)=q(L, t)$, corresponding to Eq. (20) and depicted in Fig. 6a, are seeded with multiplicative random error $\left(\epsilon=10 \%\right.$ error magnitude or error level, $\xi_{i}=$ ith random deviate) to yield "measured" outflows $q_{\text {out } \mid \epsilon}(t)$ as follows:

$q_{\mid \epsilon}(L, t)=q_{\text {out } \mid \epsilon}(t)=q_{\text {out }}(t)\left(1+\epsilon \xi_{i}\right)$

This test highlights difficulties entailed in reverse routing. As Fig. 6b shows, reverse integration after only a few spatial steps (number depends on grid resolution) amplified the errors greatly, noise gradually dominating the computed hydrograph. Hence, some sort of data conditioning is needed to control noise amplification and glean true from spurious information. To this end, we alternated routing and filtering. Naïve three-point moving average gave erratic results, from failure to control noise (perturbations persisted) to overdamping. In contrast, the symmetric, second order, five-point Savitzky-Golay low-pass filter [weights $\omega_{o}=0.486, \omega_{-1}=$ $\omega_{1}=0.343, \omega_{-2}=\omega_{2}=-0.086$, (Press et al., 1996)], Fig. 7,

$\left\langle q_{\mathrm{out}, i}\right\rangle=\sum_{j=-2}^{2} \omega_{j} q_{\mathrm{out}, i+j}$

produced well-timed and smooth, but slightly attenuated inflow signals, with mass error $\mathrm{E}_{\mathrm{M}} \leq 0.07$; negative values were eliminated before and after filtering. A four-point filter was
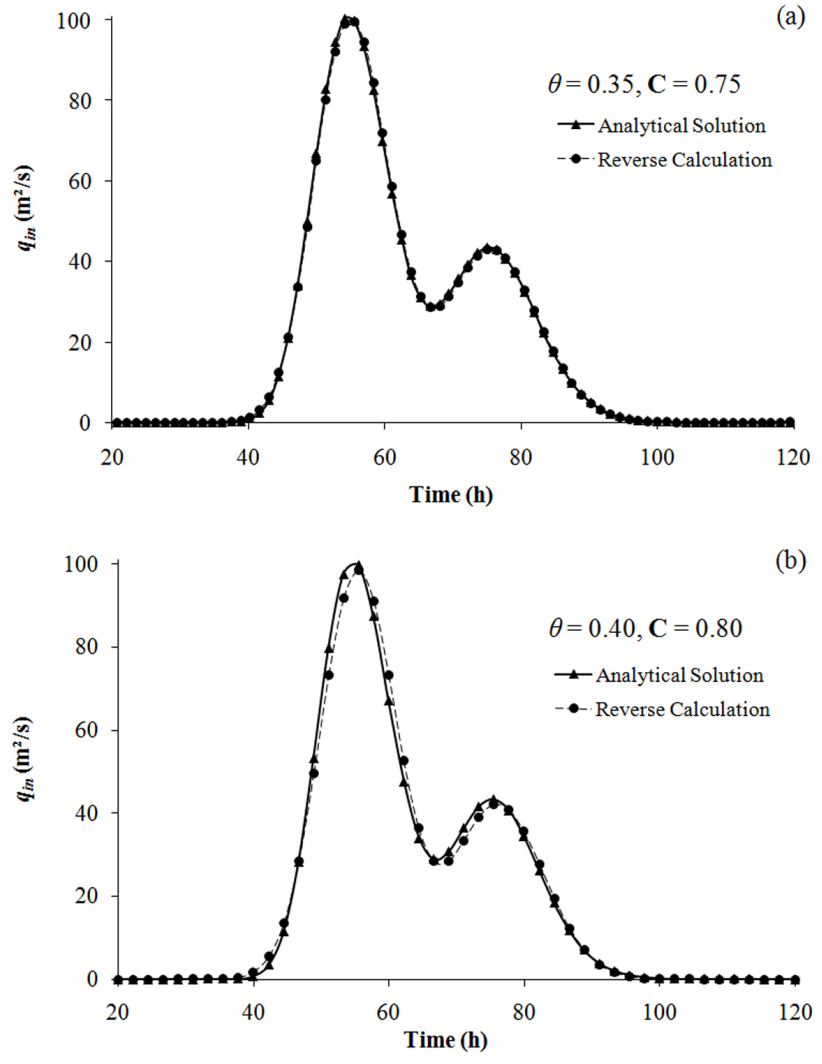

Fig. 5. Double-peak inflow hydrograph recovered through reverse routing from perfect outflow data at $x=50 \mathrm{~km}$ with grids: $\mathbf{C}=0.75$, $\theta=0.35$ (near-optimal) and $\mathbf{C}=0.8, \theta=0.4$ (non-optimal); the analytical inflow is also shown for comparison.

applied at $t=\Delta t$ and no filter at $t=0$. Figure 8 shows recovered inflows, rescaled to proper mass. Despite the somewhat lacking peak sharpness of the recovered signals, these tests indicate that this simple data conditioning retains much of the physical information intact.

Next, we use the finite-duration square pulse of Neupauer et al. (2000) to demonstrate the recovery of a source signal by reverse routing of profile field data, i.e., from observations (here, of concentration) along the stream at a constant time. In their paper, Neupauer et al. (2000) seeded that field profile with multiplicative random error $\epsilon=0.05=5 \%$ for compatibility with the comparison of the methods of Tikhonov regularisation and of minimum relative entropy. Figure $9 \mathrm{a}$ shows the field profile generated for our test, also with $\epsilon=0.05$. The unit-size square pulse shown in Fig. $9 \mathrm{~b}$ and $\mathrm{c}$ starts at $t=125$ and ends at $t=225$, has mass $M=100$ and the field profile is observed along $0 \leq x \leq 300$ at $t=T=300$; the transport parameters are $u=1$ and $D_{L}=1$, all given in consistent units. Reverse-time integration results with SavitzkyGolay filtering on a grid $\theta=0.33, \mathbf{C}=1.16$ are displayed in Fig. 9b; the recovered source signal has $r=0.25$. Note 

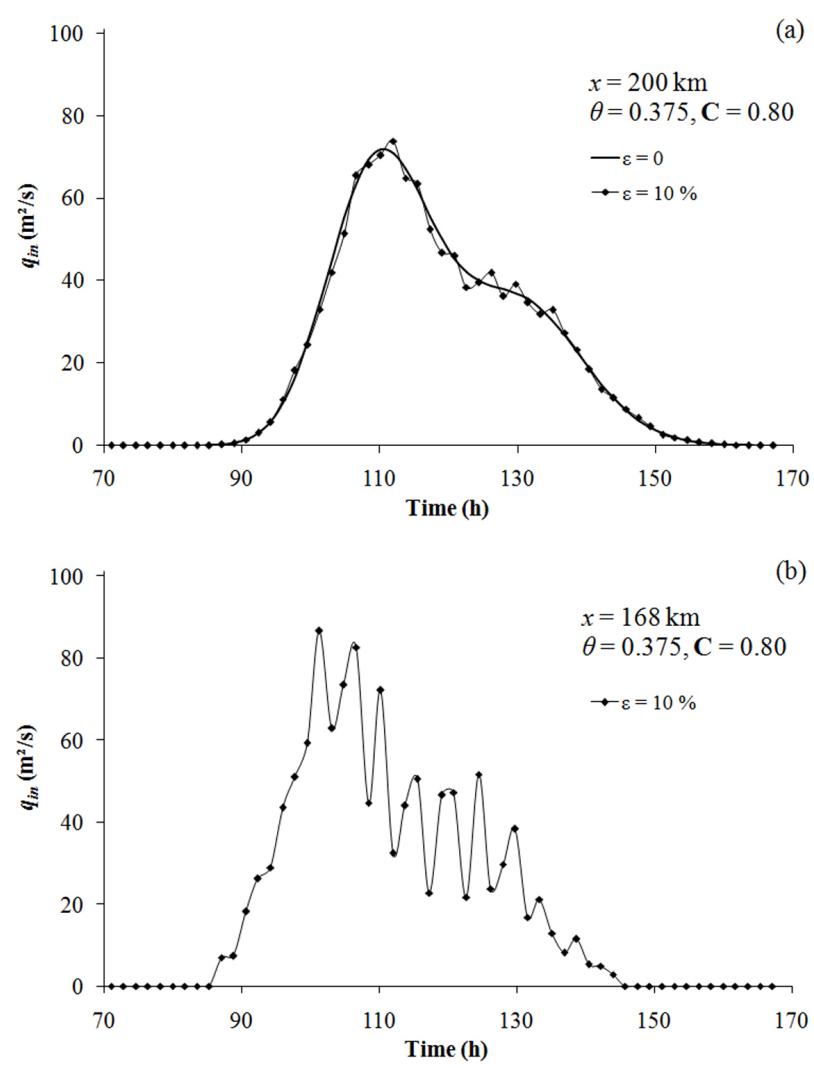

Fig. 6. (a) Field measurements of outflow hydrograph, "perfect", $\epsilon=0 \%$, and seeded with error $\epsilon=10 \%$; (b) reverse-computed discharge hydrograph after a few space steps.

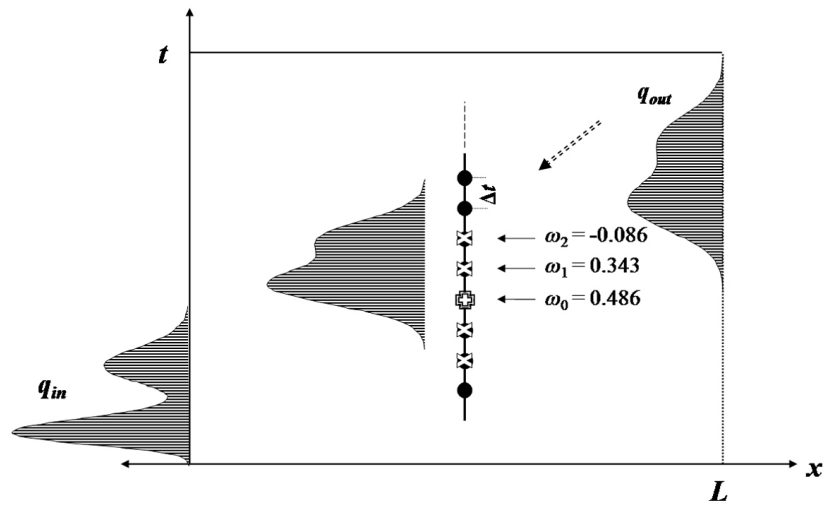

Fig. 7. Concept of reverse routing with filtering with the symmetric, second order, five-point Savitzky-Golay low-pass filter; weights: $\omega_{0}=0.486, \omega_{-1}=\omega_{1}=0.343, \omega_{-2}=\omega_{2}=-0.086$.

that data conditioning, via filtering, has enlarged the Courant number range relative to that shown in Fig. 3.
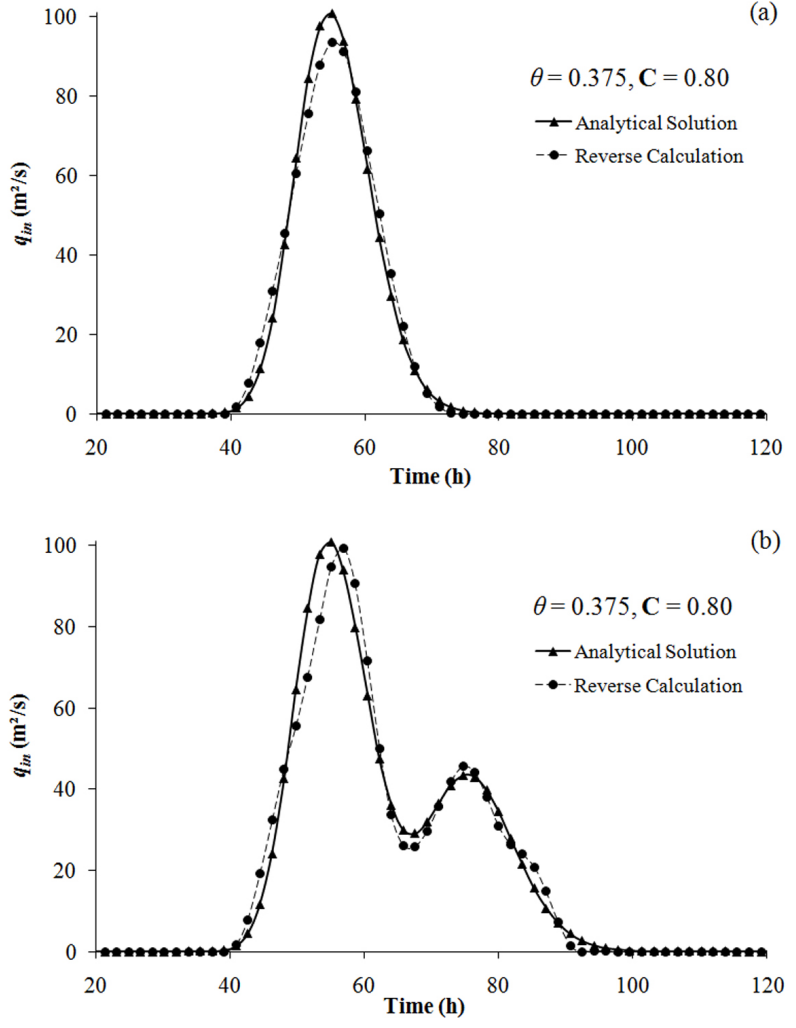

Fig. 8. Reverse routing with low-pass filtering (symmetric, second order, five-point Savitzky-Golay filter) of single-peak and doublepeak imperfect outflow hydrographs.

\section{Reverse routing of imperfect data with optimisation}

For yet sharper signal definition, we explore coupling reverse routing with optimisation, choosing the general-purpose code Solver bundled with the widely used MS Excel. Of course, any of the numerous available optimisation codes can be used instead of Solver; we stress that we used Solver simply because of its ready availability. However, because "Solver has a marked tendency to stop at a point that is not a solution and declare that it has found a solution" (McCullough and Wilson, 2002), we re-checked its solutions.

The solution was optimised using as objective function

$\min \left\{\alpha^{2} \Delta \mathrm{x} \sum(\Delta 2)^{2}+\sum\left(\delta_{\mathrm{N}}\right)^{2}\right\}$,

and the physically plausible constraints of non-negative concentrations and of the volume (or mass) of iterated hydro/polluto-graphs, or profiles, not exceeding the volume (or mass) of the data curve at the previous time step. $\sum(\Delta 2)^{2}=\sum\left\{\left[\Delta^{2}\left(F / F_{\max }\right) / \Delta x^{2}\right]_{i}\right\}^{2}$ is the sum of the squared second derivatives of a field curve $F$ (discharge or concentration) normalised by the field curve's maximum; $\sum\left(\delta_{N}\right)^{2}=\sum\left[\left(F_{i} f_{i}\right) / F_{\max }\right]^{2}$ is the sum of normalised deviations of the computed from a reference curve $f_{i}$, and $\alpha$ a 

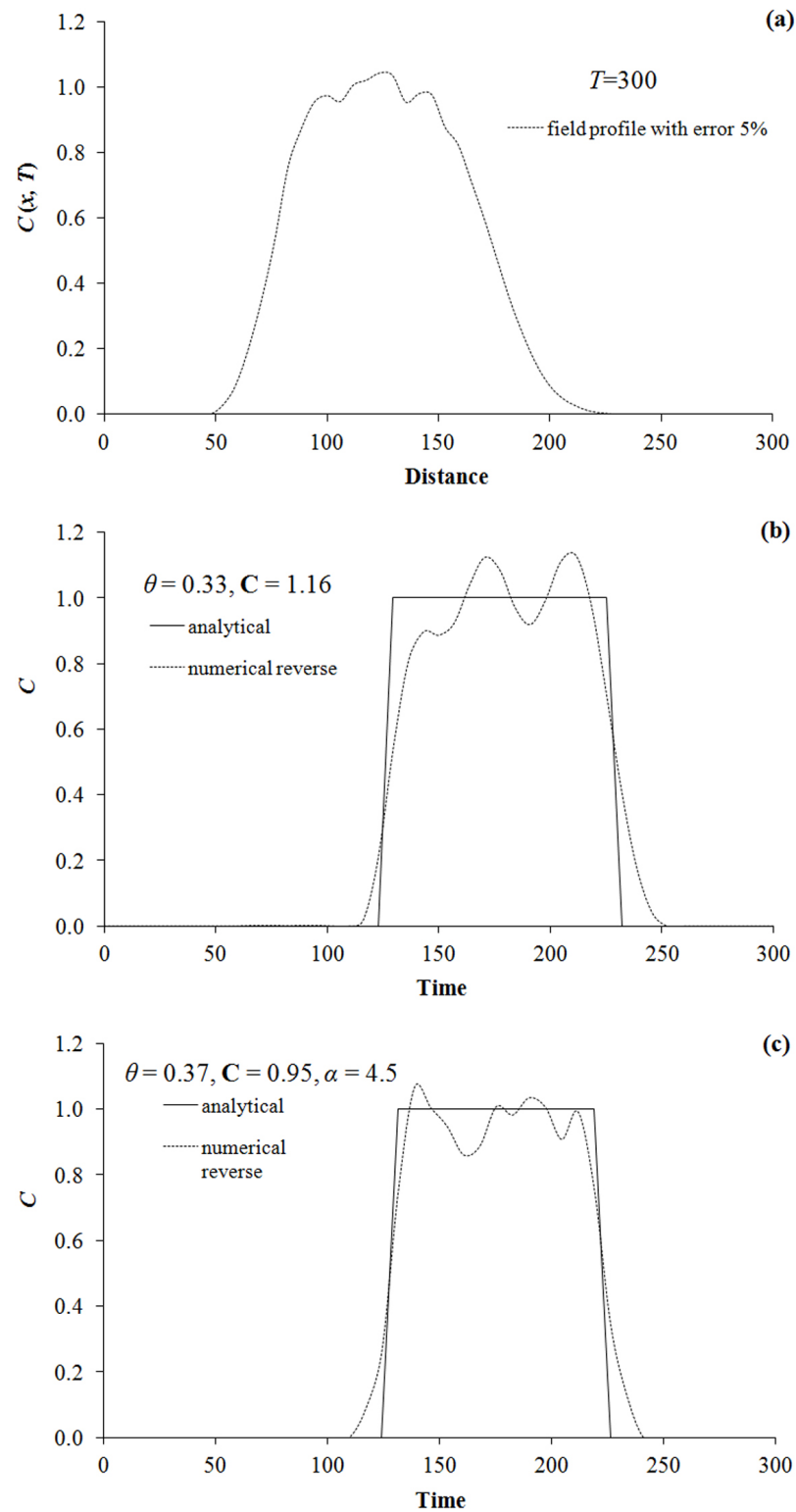

Fig. 9. Reverse routing of a concentration profile seeded with $5 \%$ multiplicative random error: (a) error-contaminated field data at time $t=T=300$; (b) recovered square pulse by reverse-routing to the source, with low-pass filtering; (c) recovered square pulse by reverse-routing to the source, with optimisation.

weighting parameter. Deviations are normalised to emphasise the peak region. Minimising $\sum(\Delta 2)^{2}$ controls noise, for stability, via a suitable $\alpha$ that balances noise suppression and artificial smoothing. Spurious oscillations are thus eliminated without loss of vital signal details. Through $\sum\left(\delta_{N}\right)^{2}$, the optimisation seeks to match the shape of the iterated to a reference curve, but since the reference curve is unknown (the solution is being sought), the deviations must

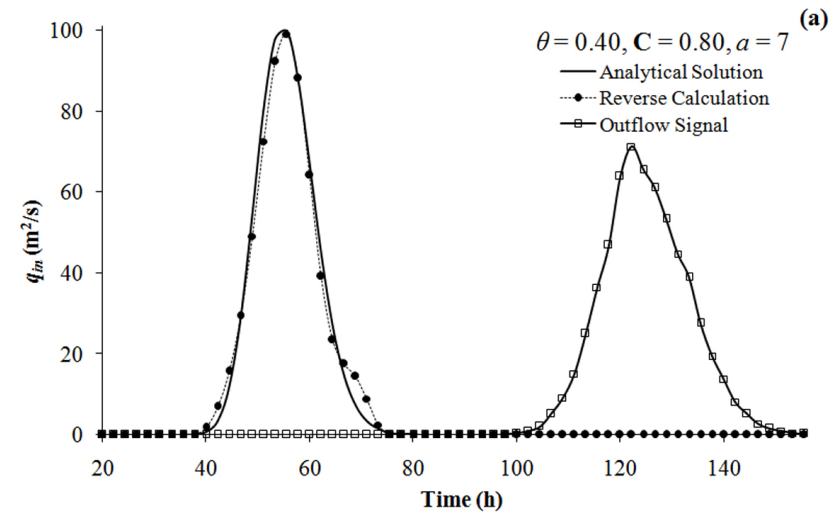

(a)

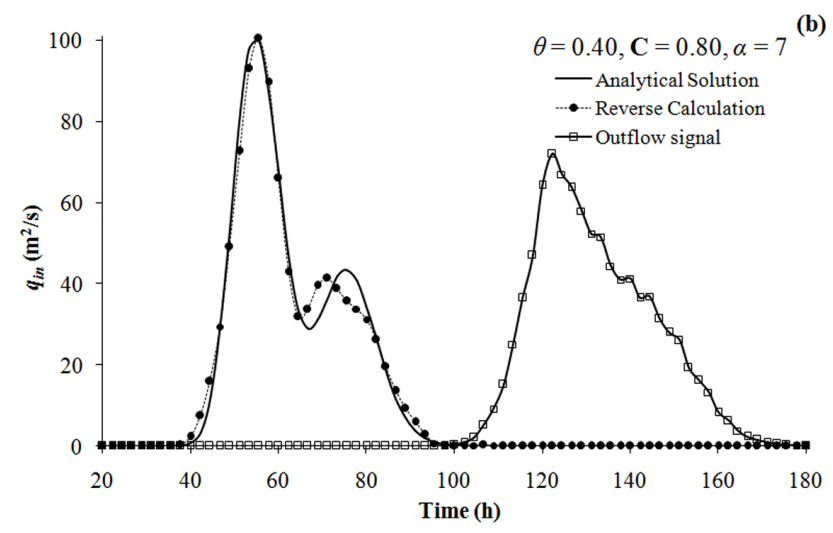

Fig. 10. Reverse flood routing with optimisation of outflow data seeded with $10 \%$-error: (a) single-peaked signal, (b) doublepeaked signal.

be referenced approximately. Arguing that routing reversal with Eq. (14) over a single grid box yields a solution whose gross shape is close to the correct one (noise affecting only the solution details), we reference deviations to the curve (hydro/polluto-graph or profile) reversed with Eq. (14).

In reverse routing with Solver optimisation, the same random error as before was added to the field data, i.e., $\epsilon=0.1=$ $10 \%$ to the single- and double-peaked source signals and $\epsilon=$ $0.05=5 \%$ to the square pulse. All runs used field data sampled at various resolutions from the same data series. Recovered source signals are rescaled to correct mass errors $E_{M} \leq$ 0.05 . For the particular signals, grids $0.25 \leq \theta \leq 0.41,0.55$ $\leq \mathbf{C} \leq 1.5$ with $\alpha$ in the range $4.0 \leq \alpha \leq 7.0$ give $r \leq 0.35$. Figure 10 shows signals optimised with $\alpha=7$ for $\theta=0.4$ and $\mathbf{C}=0.8$. The best recovered double-peaked source signal has $r=0.16$ and was obtained with $\theta=0.27, \mathbf{C}=0.75$ and $\alpha=4.5$.

Results of the square-pulse reverse transport, with Solver optimisation, shown in Fig. 9c, were computed with $\theta=0.37$, $\mathbf{C}=0.95$ and $\alpha=4.5$. Reverse routing with optimisation is again superior $(r=0.20)$ to reverse routing with lowpass filtering $(r=0.25)$, both methods achieving accuracy 
comparable to that of the Tikhonov regularisation and the minimum relative entropy methods (Neupauer et al., 2000). Relative to low-pass filtering, optimisation also improves grid design flexibility $(0.25 \leq \theta \leq 0.41$; for highest accuracy, $\theta \geq 1 / 3, \mathbf{C} \geq 2 / 3$ and mostly close to $\mathbf{C}=1 ; 4.5 \leq \alpha \leq 6.0$ ), however computing intensity increases greatly.

Based on tests with analytical solutions, our work proved the ability of the direct reverse routing scheme Eqs. (14)-(15) to recover known source signals, in conjunction with a procedure for noise control. In real applications, however, unknown source signals must be recovered from data measured in the field. Therefore, we amend the previously presented tools and synthesise the following methodology that is appropriate for real applications. From the measured field data, source signals are reverse-computed with optimisation for a series of $\alpha$-values (say, in the range $3.5 \leq \alpha \leq 6.0$ ). Then, forward routing is executed (Eqs. 6-7), using the recovered source signals as input and obtaining solutions $F_{\alpha}(x, t ; \alpha)$. These are compared to the observed field data on the basis of an error measure, such as the root-mean-squared-error (rmse), and the optimal $\alpha$ is estimated from the minimum of the rmse $\left[F_{\alpha}(x, t ; \alpha)\right]$ curve. Finally, the best estimate of the source signal is reverse-computed with the optimal $\alpha$.

\section{The reverse routing example of Bruen and Dooge}

Bruen and Dooge (2007) studied, by Fourier analysis, the stability of reverse routing with the de St. Venant equations, discretised via the four-point box scheme of Preissman, similar to the one used by Szymkiewicz $(1993,1996)$. They considered three inputs, a short-duration square pulse, a single sinusoid and a train of 10 such sinusoids, with $1 \mathrm{~h}$ and $10 \mathrm{~h}$ periods of both the pulse and the sinusoid. The base flow was $q_{\text {base }}=500 \mathrm{~m}^{3} \mathrm{~s}^{-1}$ and peak inflow $4500 \mathrm{~m}^{3} \mathrm{~s}^{-1}$; the channel had rectangular cross-sections of width $B=100 \mathrm{~m}$, slope $S_{o}=0.971 \times 10^{-3}$, Manning's $n=0.025$, and length $200 \mathrm{~km}$, but the routing was executed only over the first $100 \mathrm{~km}$. Reverse routing was unable to recover the high-frequency inflow signals, the most severe of which was the single sinusoid of 1-h period; the reverse calculation either broke down rapidly or yielded inflow hydrographs with peaks of 1500 $2500 \mathrm{~m}^{3} \mathrm{~s}^{-1}$. In the case of the 10 -h sinusoid and pulse, the outcomes were quite good; the inflow signal was recovered well, except in one (sinusoid) and two intervals (pulse) of concentrated oscillations associated with rapid flow changes. Interestingly, Bruen and Dooge found that the stability of reverse routing increased the closer to the outflow section of the grid element the temporal derivatives were evaluated; this is opposite from the behaviour of the reverse Muskingum box scheme, in which $\theta \rightarrow 0$ promotes instability.

The parameters of the corresponding linearised CDE (1) were evaluated from Eq. (2), $c_{k}=6.325 \mathrm{~m} \mathrm{~s}^{-1}$ by linear regression in $400 \mathrm{~m}^{3} \mathrm{~s}^{-1} \leq q \leq 6000 \mathrm{~m}^{3} \mathrm{~s}^{-1}$, and from Eq. (3), $D=11073 \mathrm{~m}^{2} \mathrm{~s}^{-1}$ at the uniform flow rate $q_{o}=2500 \mathrm{~m}^{3} \mathrm{~s}^{-1}$

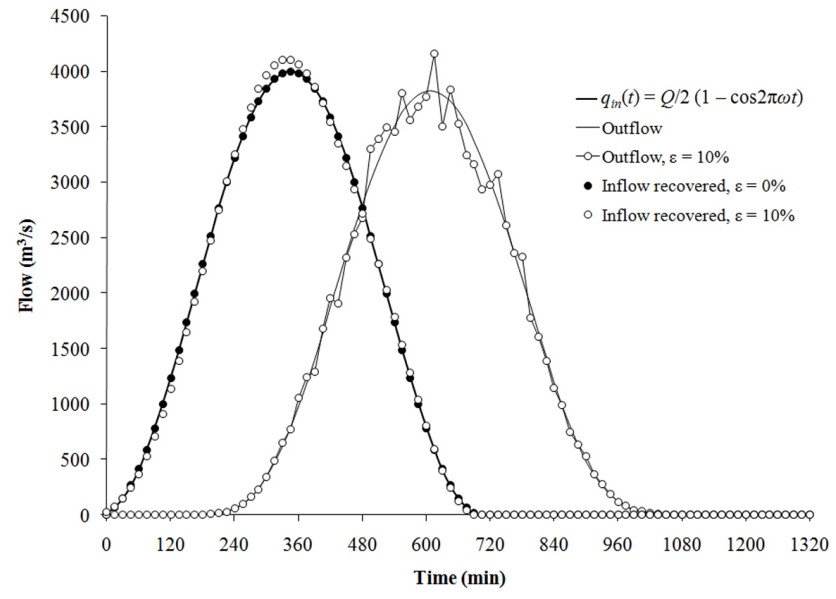

Fig. 11. Reverse routing of the sinusoidal inflow hydrograph of Bruen and Dooge (2007).

$\left(y_{o} \approx 6.28 \mathrm{~m}, \mathbf{F}_{\mathbf{0}} \approx 0.5\right)$. We considered the single sinusoid (above $q_{\text {base }}$ ) with period $T=2 \pi / \omega=10 \mathrm{~h}, q_{\text {in }}(t)=Q / 2(1-$ $\cos 2 \pi \omega t$ ), and generated the outflow hydrograph by forward routing through 15 Muskingum-reservoirs $(\Delta x=6667 \mathrm{~m}, \theta=$ $0.2374 ; \Delta t=900 \mathrm{~s}, \mathbf{C}=0.84$ ). Figure 11 shows the remarkably accurate inflow recovery attained, even when the outflow signal was seeded with $\epsilon=10 \%$ error. In the latter case, reverse routing was coupled with low-pass filtering (symmetric, second order, eleven-point Savitzky-Golay filter, with weights: $\omega_{0}=0.207, \omega_{-1}=\omega_{1}=0.196, \omega_{-2}=\omega_{2}=0.161$, $\omega_{-3}=\omega_{3}=0.103, \omega_{-4}=\omega_{4}=0.021, \omega_{-5}=\omega_{5}=-0.084$; Press et al., 1996) and $1 \%$ mass correction. The good performance of the method on a grid with $\theta \approx 0.24$ was verified in additional tests with $\theta<0.25$ (and $\mathbf{C}>0.5$ ), in which the sinusoidal inflow hydrograph was regained largely intact. The increased flexibility in the choice of grid parameters is due to the large volume of the wave (an order of magnitude more than in the previous tests), given the similar number of space and time steps involved in the reverse routing. Generally, it appears that the overall high fidelity of the recovery benefits from the simplicity of the reverse routing scheme, which endows it with numerical robustness.

The 15-Muskingum-reservoirs outflow hydrograph is very close to that of the Kalinin-Miljukov model's 29 concentrated $(\theta=0)$ linear reservoirs (each representing a unit reach $L_{\mathrm{KM}} \approx 3450 \mathrm{~m} ; \theta=0$ ), the highest spatial resolution possible in storage routing with $\theta \geq 0$ (Kalinin and Miljukov, 1958; Koussis, 2009). Using $\Delta x<L_{\mathrm{KM}}$ is possible, but then $\theta<0$, implying the physical abnormality that storage declines while the inflow rises. Reversing the Kalinin-Miljukov outflow using, by necessity, fewer storage elements than 29 understandably yields slightly less accurate inflows, because the forward and reverse schemes are not compatible. 


\section{Summary and conclusions}

We have shown that inflows can be identified from outflows by reverse application of the Muskingum routing scheme, which approximates diffusion-wave behaviour closely by appropriate choice of its parameters, assuming kinematic-wave as dominant mode of flood propagation (holds largely). Because routing against the wave propagation is an ill-posed problem, the reverse solution of error-seeded data was smoothness-constrained for stability.

In the case of perfect data, theoretically constraining the coefficients of the reverse routing scheme assisted in error control; however, optimal grid design was derived through numerical experimentation against exact solutions of the convection-diffusion equation, for single and composite waves. Wave propagation was backtracked well in all instances for a range of grid parameters. In an arduous square pulse test, results comparable to those of more complex methods were achieved. Seeding the outflow signal with random errors (mimicking field observations) made reverse routing unstable. To cope with spurious oscillations, the reversed solution was conditioned (smoothing) via low-pass filtering or optimisation; good-quality inflow hydrographs were recovered by either method, but the computationally demanding optimisation was superior. Also advanced was an optimisation-based procedure to identify an unknown signal from imperfect data, involving multiple reverse and forward runs; an objective comparison of predicted vs. the observed outflow hydrograph determines the optimal $\alpha$-value for the identification of the inflow signal by reverse routing. Finally, the reverse Muskingum routing scheme performed as well as the orders of magnitude more demanding reverse solution of the St. Venant equations of flood wave motion.

Our tests involved prismatic channels to benefit from existing analytical solutions, but the reverse routing scheme is also applicable to natural streams, with properly selected grids, as is the forward Muskingum scheme. Because most stream morphologies prescribe variable space steps (by numerical analysis: accuracy always diminishes on variable grids), $\Delta t$ must be chosen such that the $\mathbf{C}$-values are an acceptable compromise over the $\theta(\Delta x)$-range.

This study leads us to conclude that the good fidelity of inflow identification rests on the simplicity of the Muskingum storage reverse routing scheme that endows it with numerical robustness and computational efficiency.

Edited by: A. Bartzokas

Reviewed by: two anonymous referees

\section{References}

Atmadja, J. and Bogtzoglou, A. C.: Pollution source identification in heterogeneous porous media, Water Resour. Res., 37, 2113 2125, 2001.

Bowen, J. D., Koussis, A. D., and Zimmer, D. T.: Storm drain design - diffusive flood routing for PCs, J. Hydraul. Eng., 115, 1135-1150, 1989.

Bronstein, I. N. and Semendjajew, K. A.: Taschenbuch der Mathematik, 412 pp., Verlag Harri Deutsch, Zürich, 1964.

Bruen, M. and Dooge, J. C. I.: Harmonic analysis of the stability of reverse routing in channels, Hydrol. Earth Syst. Sci., 11, 559568, doi:10.5194/hess-11-559-2007, 2007.

Cunge, J. A.: On the subject of a flood propagation computation method (Muskingum method), J. Hydraul. Res., 7, 205-230, 1969.

Dooge, J. C. I.: Linear theory of hydrologic systems, Tech. Bull. No. 1468, Agricultural Research Service, United States Department of Agriculture, Washington D.C., 1973.

Fischer, H. B., List, E. J., Koh, R. C. Y., Imberger, J., and Brooks, N. M.: Mixing in inland and coastal waters, Academic Press, 1979.

García-Delgado, R. A. and Koussis, A. D.: Groundwater solute transport with hydro-geochemical reactions, Ground Water, 35, 243-249, 1997.

Kabala, Z. J. and Skaggs, T. H.: Comment on "Minimum relative entropy inversion: Theory and application of recovering the release history of a groundwater contaminant", by A. D. Woodbury and T. J. Ulrych, Water Resour. Res., 34, 2077-2079, 1998.

Kalinin, G. P. and Miljukov, P. I.: Approximate methods for computing unsteady flow movement of water masses (in Russian), Transactions Central Forecasting Institute, 66, 1958.

Koussis, A.: Ein Verbessertes Näherungsverfahren zur Berechnung von Hochwasserabläufen [An improved approximate flood routing method (in German)]. TB no. 15, Institut für Hydraulik und Hydrologie, Technische Hochschule Darmstadt, Germany, 1975.

Koussis, A. D.: Theoretical Estimation of Flood Routing Parameters, J. Hydraul. Div. ASCE 104(HY1), 109-115, 1978.

Koussis, A. D.: Comparison of Muskingum Method Difference Schemes, J. Hydraul. Div., ASCE, 106(HY5), 925-929, 1980.

Koussis, A. D., Saenz, M. A., and Tollis, I. G.: Pollution routing in streams, J. Hydraul. Div. ASCE 109(HY12), 1636-1651, 1983.

Koussis, A. D.: Unified theory for flood and pollution routing, J. Hydraul. Div. ASCE 109(HY12), 1652-1664, 1983.

Koussis, A. D., Kokitkar, P., and Mehta, A.: Modeling DO conditions in streams with dispersion, J. Env. Eng. ASCE, 116, 601614, 1990.

Koussis, A. D., Pesmajoglou, S., and Syriopoulou, D.: Modelling biodegradation of hydrocarbons in aquifers: When is the use of the instantaneous reaction approximation justified?, J. Contam. Hydrol., 60, 287-305, 2003.

Koussis, A. D.: An assessment review of the hydraulics of storage flood routing 70 years after the presentation of the Muskingum method, Hydro. Sci. J., 54, 43-61, 2009.

Koussis, A. D.: Reply to the Comment of M. Perumal on An assessment review of the hydraulics of storage flood routing 70 years after the presentation of the Muskingum method, Hydrolog. Sci. J., 55, 1431-1441, 2010.

Kreft, A. and Zuber, A.: On the physical meaning of the dispersion equation and its solutions for different initial and boundary conditions, Chem. Eng. Sci., 33, 1471-1480, 1978. 
Li, W. H.: Effect of dispersion in DO-sag in uniform flow, J. San. Eng., 98(SA1); (1973) Discussions in J. Env. Eng. 99(EE2), 1972.

Lighthill, M. J. and Whitham, G. B.: On kinematic waves I. Flood movement in long rivers, P. Roy. Soc. Lond. A Mat., 229, 281316, 1955.

Liu, C. and Ball, W. P.: Application of inverse methods to contaminant source identification from aquitard diffusion profiles at Dover, AFB, Delaware, Water Resour. Res., 35, 1975-1985, 1999.

McCullough, B. D. and Wilson, B.: On the accuracy of statistical procedures in Microsoft Excel 2000 and Excel XP, Comput. Stat. Data Anal., 40, 713-721, 2002.

Michalak, A. M. and Kitanidis, P. K.: Estimation of historical groundwater contaminant distribution usin an adjoint state method applied to geostatistical inverse modelling, Water Resour. Res., 40, W08302, doi:10.1029/2004WR003214, 2004.

Nash, J. E.: A Note on the Muskingum Flood-Routing Method, J. Geophys. Res., 64, 1053-1056, 1959.

Neupauer, R. M., Borchers, B., and Wilson, J. L.: Comparison of inverse methods for reconstructing the release history of groundwater contamination source, Water Resour. Res., 36, 2469-2475, 2000.

Perkins, S. P. and Koussis, A. D.: A stream-aquifer interaction model with diffusive wave routing, J. Hydraul. Eng., 122(4), 210-219, 1996.

Perumal, M. and Sahoo, B.: Applicability criteria of the variable parameter Muskingum stage and discharge routing methods. Water Resour. Res., 43, 1-20, W05409, doi:10.1029/2006WR004909, 2005.

Press, W. H., Teukolsky, S. A., Vetterling, W. T., and Flannery, B. P.: Numerical Recipes in FORTRAN 77: The Art of Scientific Computing, Cambridge University Press, 1996.

Seus, G. J. and Rösl, G.: Hydrologische Verfahren zur Berechnung des Hochwasserwellenablaufs in Flüssen, in: Elektronische Berechnung von Rohr- und Gerinneströmungen, edited by: W. Zielke, Erich Schmidt Verlag, 1972.
Skaggs, T. H. and Kabala, Z. J.: Recovering the release history of a groundwater contaminant, Water Resour. Res., 30, 71-79, 1994.

Skaggs, T. H. and Kabala, Z. J.: Recovering the history of a groundwater contaminant plume: Method of quasi-reversibility, Water Resour. Res., 31, 2669-2673, 1995.

Skaggs, T. H. and Kabala, Z. J.: Limitations in recovering the history of a groundwater contaminant plume, J. Contam. Hydrol., 33, 347-359, 1998.

Snodgrass, M. F. and Kitanidis, P. K.: A geostatistical approach to contaminant source identification, Water Resour. Res., 33, 537 546, 1997.

Syriopoulou, D. and Koussis, A.D.: 2-D modeling of advectiondominated solute transport in groundwater by the matched artificial dispersivity method, Water Resour. Res., 27, 865-872, 1991.

Szöllósi - Nagy, A.: Input detection by the discrete linear cascade model, J. Hydrol., 89, 353-370, 1987.

Szymkiewicz, R.: Solution of the inverse problem for the Saint Venant equations, J. Hydrol., 147, 105-120, 1993.

Szymkiewicz, R.: Numerical stability of the implicit four points scheme applied to inverse linear flood routing, J. Hydrol., 176, 13-23, 1996.

Szymkiewicz, R.: An alternative IUH for the hydrological lumped models, J. Hydrol., 259, 246-253, 2002.

Venetis, C.: The IUH of the Muskingum channel reach, J. Hydrol. 7, 444-447, 1969.

Weinmann, P. E.: Comparison of flood routing methods in natural rivers. Report no. 2/1977. Dept Civil Engng, Monash University, Clayton, Victoria, Australia, 1977.

Weinmann, P. E. and Laurenson, E. M.: Approximate flood routing methods: a review, J. Hydraul. Div. ASCE 105(12), 1521-1536, 1979.

Woodbury, A. D. and Ulrich, T. J.: Minimum relative entropy inversion: Theory and application of recovering the release history of a groundwater contaminant, Water Resour. Res., 32, 2671-2681, 1996.

Woodbury, A. D. and Ulrich, T. J.: Reply, Water Resour. Res., 34, 2081-2084, 1998. 